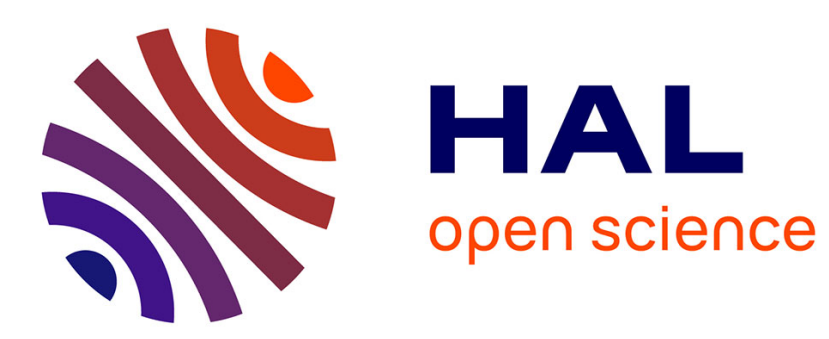

\title{
Les paradoxes de la géographie des Lumières"
}

\author{
Isabelle Laboulais
}

\section{To cite this version:}

Isabelle Laboulais. Les paradoxes de la géographie des Lumières". La construction des savoirs (XVIIIe-XIXe siècles), Presses universitaires de Lyon, pp.139-158, 2009, 10.4000/books.pul.11825 . hal-02991312

\section{HAL Id: hal-02991312 \\ https://hal.science/hal-02991312}

Submitted on 5 Nov 2020

HAL is a multi-disciplinary open access archive for the deposit and dissemination of scientific research documents, whether they are published or not. The documents may come from teaching and research institutions in France or abroad, or from public or private research centers.
L'archive ouverte pluridisciplinaire HAL, est destinée au dépôt et à la diffusion de documents scientifiques de niveau recherche, publiés ou non, émanant des établissements d'enseignement et de recherche français ou étrangers, des laboratoires publics ou privés. 
Isabelle Laboulais, «Les paradoxes de la géographie des Lumières », dans Lise Andries (dir.), La construction des savoirs (XVIIIe-XIXe siècles), Lyon, Presses Universitaires de Lyon, 2009, p. 139-158.

\title{
Les paradoxes de la géographie des Lumières 1
}

\author{
Isabelle Laboulais (Université Marc Bloch, Strasbourg 2)
}

En 1808, dans les Annales des voyages, de la géographie et de l'histoire, alors qu'il décrivait la situation dans laquelle se trouvait la géographie, Malte-Brun notait avec amertume : «Il faut pourtant l'avouer : ces nobles et intéressantes études ne brillent point en France d'un aussi vif éclat que les autres sciences.»2; puis, en 1810, dans la préface de son Précis de géographie universelle, il poursuivait son état des lieux de la géographie et ajoutait : « La jeunesse la redoute, les savants la négligent, les gens du monde la dédaignent »3. Ce constat peut a priori surprendre quand on songe notamment aux travaux de Sergio Moravia4 et de Numa Broc5 qui ont montré la position décisive occupée par les savoirs géographiques dans l'édition et dans la culture savante de la France des Lumières6. Cependant, si paradoxal qu'il puisse paraître, force est de constater que le constat dressé par Malte-Brun reflète avec une grande lucidité le manque de visibilité de la géographie dans les instances de savoir de la fin du XVIIIe et du début du XIXe siècles.

1 La contribution proposée ici a été présentée le 13 novembre 2003 au séminaire de Lise Andries, elle correspond à une première étape de ma contribution à l'introduction du volume collectif qu'Hélène Blais et moi-même avons dirigé: Géographies plurielles. Les sciences géographiques au moment de l'émergence des sciences humaines (1750-1850), L'Harmattan, Histoire des sciences humaines, 2006. La version complète de cette introduction intitulée «Les figures de la géographie moderne. Fragmentations et régularités » (p. 9-59) a été co-écrite avec Hélène Blais.

2 MALTE-BRUN (Conrad), Annales des voyages, de la géographie et de l'histoire, ou Collection des voyages nouveaux les plus estimés, traduits de toutes les langues européennes ; des relations originales, inédites, communiquées par des voyageurs français et étrangers, tome premier, Paris, 1808, p. 6-7. Toutes les citations respectent l'orthographe originale.

3 MALTE-BRUN (Conrad), Précis de la géographie universelle ou Description de toutes les parties du monde sur un plan nouveau, d'après les grandes divisions naturelles du globe, tome premier, Histoire de la géographie, Paris, 1810 , p. 5.

4 MORAVIA (Sergio), "Philosophie et géographie à la fin du XVIIIe siècle », Studies on Voltaire and the Eighteenth Century, 1967, n 57, p. 937-1011.

5 BROC (Numa), La géographie des philosophes. Géographes et voyageurs français au XVIIIe siècle, Paris, Ophrys, 1975.

6 Déjà en 1836, Humboldt avait, à sa manière, relevé l'adéquation de la géographie avec l'épistémè de la fin du XVIIIe siècle. Dans son Examen critique de l'histoire de la géographie du nouveau continent et des progrès de l'astronomie nautique aux XVe et XVIe siècles il notait en effet que l'« on pourrait être tenté d'admettre que ces étonnantes découvertes qui se secondaient pour ainsi dire mutuellement, que ces doubles conquêtes dans un monde physique et dans le monde intellectuel n'eussent été dignement appréciées de nos jours que dans un siècle où l'histoire de la civilisation humaine a été tracée par des philosophes qui pouvaient embrasser d'un seul coup d'œil les progrès de la géographie astronomique et physique, de l'art du navigateur, de la botanique et de la zoologie descriptives ». Tout en reprenant les deux dimensions -mathématique et descriptive- de la géographie, Humboldt relie ce champ du savoir à une connaissance du monde qu'il juge caractéristique de l'Europe des Lumières. HUMBOLDT (Alexandre de), Examen critique de l'histoire de la géographie du nouveau continent et des progrès de l'astronomie nautique aux XVe et XVIe siècles, tome I et II, Paris, Librairie Gide, 1836, p. 3. 
Isabelle Laboulais, «Les paradoxes de la géographie des Lumières », dans Lise Andries (dir.), La construction des savoirs (XVIIIe-XIXe siècles), Lyon, Presses Universitaires de Lyon, 2009, p. 139-158.

\section{Le manque de visibilité institutionnelle de la géographie}

Déjà, en 1782, dans son «Discours sur la géographie » publié en tête des volumes de l'Encyclopédie méthodique, Masson de Morvilliers s'était désolé de l'indifférence manifestée pour cette science dans les maisons d'instruction. Il mentionnait toutefois l'exception que constituait le projet de l'Abbé Grenet qui avait proposé de « joindre la géographie à l'éducation »7. Mais, à cette exception près, sa position restait très marginale dans les disciplines enseignées8. Certes, des cours de géographie étaient dispensés dans les collèges jésuites - cours qui faisaient coexister la tradition mathématique et la tradition littéraire de la géographie-, mais, pour constater l'émergence d'un enseignement de géographie à part entière, il fallut attendre les institutions mises en place pendant et après la Révolution. A partir de 1789, et plus encore de 1792, une série de réformes a en effet contribué à rénover les institutions scolaires et la géographie a plutôt bénéficié de ce mouvement9. Pendant la Révolution, outre qu'un enseignement de géographie a été instauré au sein de l'éphémère Ecole Normale de l'an III 10, des cours ont également été dispensés dans les écoles centrales11 ainsi qu'à l'Ecole des Mines12. Par ailleurs, en 1801, la géographie bénéficia d'une place spécifique en horaires et en programmes dans le cadre de la nouvelle université, mais dès 1821 elle perdit cette place et ne fit plus l'objet de cours qu'en classe

7 MASSON DE MORVILLIERS (Nicolas), Encyclopédie méthodique, Géographie moderne, tome I, Paris, 1782, p. XVII.

8 DAINVILLE (François de), «Enseignement des «géographes » et «géomètres » », in TATON (René) (dir.), Enseignement et diffusion des sciences en France au XVIIIe siècle, Paris, Hermann, 1964, p. 481-492.

9 BRET (Patrice), DORIGNY (Marcel) (dir.), Sciences et techniques autour de la Révolution française, Journées d'études des 10 et 11 décembre 1999, Cité des sciences et de l'industrie - Monnaie de Paris, in Annales de la Révolution française, 2000, p. 320.

10 NORDMAN (Daniel) (dir.), L'Ecole normale de l'an III. Leçons d'histoire, de géographie, d'économie politique, Paris, Dunod, 1994.

11 Bien que la géographie n'apparaisse pas parmi les neuf cours initialement institués dans les écoles centrales (le dessin, l'histoire naturelle et les langues anciennes composaient la première section ; les mathématiques, la physique et la chimie composaient la deuxième; la grammaire générale, l'histoire, les belles lettres et la législation composaient la troisième), elle fut enseignée dans plusieurs d'entre elles. Des éléments de géographie physique enrichissaient les leçons d'histoire naturelle et des notions de géographie politique et historique complétaient les cours d'histoire. Cf. COMPERE (Marie-Madeleine), «La question des disciplines scolaires dans les Ecoles centrales. Le cas des langues anciennes », Histoire de l'éducation "Les enfants de la patrie. Education et Enseignement sous la Révolution française », 1989, $\mathrm{n}^{\circ}$ 42, p. 139-181; GUY (Marcel), "L'enseignement de l'histoire dans les écoles centrales (an IV - an XII) », Annales historiques de la Révolution française, 1981, $\mathrm{n}^{\circ}$ 243, $\mathrm{p}$. 89-122 ; TRENARD (Louis), «Les écoles centrales », Dix-Huitième siècle « Au tournant des Lumières », 1982, $\mathrm{n}^{\circ}$ 14 , p. 57-74.

12 L'Arrêté de l'Agence des mines relatif aux cours de la maison d'instruction (approuvé le 18 brumaire an III) qui fut publié dans le numéro deux du Journal des Mines annonçait quatre cours publics et gratuits dispensés à partir de frimaire. Parmi eux, figurait un cours de minéralogie et de géographie physique dispensé par Hassenfratz. On pourra 
Isabelle Laboulais, «Les paradoxes de la géographie des Lumières », dans Lise Andries (dir.), La construction des savoirs (XVIIIe-XIXe siècles), Lyon, Presses Universitaires de Lyon, 2009, p. 139-158.

de huitième et de septième 13 ; quant au projet napoléonien d'une Ecole de la géographie au Collège de France il ne vit jamais le jour14. Malgré son intrusion au sein de quelques institutions scolaires -aux existences souvent brèves-, la géographie a peiné à s’imposer parmi les disciplines enseignées et ce retard reflète assez fidèlement le manque d'instances géographiques caractéristique de la France au tournant des XVIIIe et XIXe siècles. D’ailleurs, dans la préface qu'il rédigea en 1808 pour le premier numéro des Annales de Voyages, Malte-Brun reliait l'absence d'une structure fédérative à l'état d'abandon dans lequel se trouvait la géographie. Il notait : «Ces efforts isolés de quelques savants demandent pour réussir un point de réunion, un centre de communications. Enfin, il est temps qu'à l'exemple de l'histoire naturelle, de la chimie et de la médecine, les sciences géographiques possèdent un dépôt où les hommes voués à ce genre d'études puissent consigner en commun des travaux qui tendent au même but. »15. On se trouve en effet là à un moment où la Société de géographie de Paris n'a pas encore été fondée elle ne le sera qu'en 182116-, et où la seule institution réunissant des géographes est le Dépôt général de la guerre. Pourtant, en aucun cas, celui-ci ne peut jouer pour la géographie le rôle qui revenait alors au Muséum pour les sciences naturelles. Certes, en 1795, le Dépôt général de la guerre devint Dépôt général de la guerre et de la géographie. Ainsi désigné, il devait offrir une formation initiale spécifiquement adaptée aux ingénieurs-géographes et, dans le même temps, réunir des savants dont la mission était de «porter au plus haut degré de développement et de gloire les sciences de l'astronomie et de la géographie »17. Cependant, le projet de Calon échoua et le Dépôt de la guerre fut réduit à n'être guère plus qu'un dépôt de cartes. Le Musée de Géographie, de topographie militaire et d'hydrographie qu'il avait imaginé fut un projet qui, lui

aussi consulter BIREMBAUT (A.), «L'enseignement de la minéralogie et des techniques minières », in TATON (René) (dir.), Enseignement et diffusion des sciences en France au XVIIIe siècle, op. cit., p. 365-418.

13 LEFORT (Isabelle), La Lettre et l'esprit, géographie scolaire et géographie savante en France, Paris, CNRS, 1982, p. 15.

${ }_{14}$ Cette Ecole de la géographie au Collège de France était censée être un bureau central qui pourrait rassembler des informations de différents endroits dans le monde, quatre chaires avaient été envisagées : une chaire de géographie maritime, une chaire de géographie européenne, une chaire de géographie extra-européenne et une chaire de géographie commerciale et statistique. Cf. GODLEWSKA (Anne M.C.), Geography unbound. French Geographic Science from Cassini to Humboldt, Chicago, The University of Chicago Press, 1999, p. 156.

15 MALTE-BRUN (Conrad), Annales des voyages, op. cit., p. 7.

16 FIERRO (Alfred), La Société de géographie de 1821-1946, Paris, Librairie Droz-Librairie Champion, 1983 ; LEJEUNE (Dominique), Les sociétés de géographie en France et l'expansion coloniale au XIXe siècle, Paris, Albin Michel, 1993.

17 Lettre de Calon à Méchain, 13 pluviôse an III, cité dans BRET (Patrice), « Le Dépôt général de la guerre et la formation scientifique des ingénieurs-géographes militaires en France (1789-1830) », Annals of Science, 1991, n 48, p. 117. 
Isabelle Laboulais, «Les paradoxes de la géographie des Lumières », dans Lise Andries (dir.), La construction des savoirs (XVIIIe-XIXe siècles), Lyon, Presses Universitaires de Lyon, 2009, p. 139-158.

aussi, tourna court18. Enfin, l'Ecole des géographes qui fut créée en 1797, était accessible après une seule année à Polytechnique, mais devant la médiocrité de ses recrutements, elle fut fermée après la suppression du Cadastre en 1802. Ainsi donc, en 1808, le Dépôt de la guerre se consacrait avant tout à la topographie -tout comme le firent les élèves de l'Ecole impériale des ingénieurs géographes fondée quelques mois plus tard, le 30 octobre 1809-19, si bien que ni le Dépôt, ni aucune institution connexe n'étaient susceptibles de correspondre à ce que Malte-Brun nommait un point de réunion pour des hommes qui s'intéressent à la connaissance du monde.

Au sein des instances académiques, la situation de la géographie n'était guère plus favorable. En effet, jusqu'en 1793, il n'exista à l'Académie royale des Sciences aucune classe de géographie, pas même après la réorganisation de 1785 censée embrasser l'universalité des sciences. A cette date, seules l'agriculture, l'histoire naturelle, la minéralogie et la physique furent alors associées aux classes de l'Académie20. Certes, en 1726 une charge de géographe ordinaire avait été créée et attribuée à Jacques-Philippe Maraldi, mais celui-ci était astronome et cumulait donc cette charge de géographe avec sa pension d'astronome. Ce n'est qu'à sa mort, en juin 1730 que Buache lui succéda, portant cette fois le titre « d'adjoint géographe ». La géographie académique resta dans cette position subalterne pendant tout l'Ancien Régime. L'article II des statuts de 1785 n'eut en effet que peu d'incidence sur ce domaine de savoir puisqu'il stipulait simplement que « l'adjoint géographe (...) prendra, à l'avenir, le titre d'associé géographe »! Cependant, à partir de 1785, plusieurs savants liés à la géographie par des savoirs connexes, entrèrent à l'Académie des Sciences : Cassini devint associé de la classe d'astronomie, Guettard -l'auteur d'une « géographie minéralogique »- devint pensionnaire de la classe de botanique et agriculture, Desmarest -chargé de la «géographie physique» dans l'Encyclopédie et l'Encyclopédie méthodique- devint pensionnaire de la classe d'histoire naturelle et de minéralogie. Dans une étude consacrée à la présence de la géographie dans les leçons de l'Ecole Normale de l'an III, Daniel Nordman a fait

18 BROC (Numa), «Un musée de géographie en 1795 », Revue d'histoire des sciences, 1974, n 27, p. 37-43.

19 PANSINI (Valeria), "L'œil du topographe et la science de la guerre. Travail scientifique et perception militaire (1760-1820) », Thèse de l'EHESS, sous la direction de Jaques Revel, EHESS, 2002. Voir en particulier le chapitre 2. 20 En 1699, les pensionnaires de l'Académie rassemblent 3 géomètres, 3 astronomes, 3 mécaniciens, 3 anatomistes, 3 chimistes, 3 botanistes et 2 associés pour chaque classe. En 1785, on compte désormais 8 classes, contre 6 auparavant, avec pour chacune d'elles 3 pensionnaires et 3 associés : géométrie, astronomie, mécanique, physique générale, anatomie, chimie et métallurgie, botanique et agriculture, histoire naturelle et minéralogie. BRIAN (Eric), DEMEULENAERE-DOUYERE (Christiane. dir.), Histoire et mémoire de l'Académie des sciences. Guide de recherches, Paris, Lavoisier, 1996, p. 22. 
Isabelle Laboulais, «Les paradoxes de la géographie des Lumières », dans Lise Andries (dir.), La construction des savoirs (XVIIIe-XIXe siècles), Lyon, Presses Universitaires de Lyon, 2009, p. 139-158.

un constat identique qui l'a conduit à parler d'une « géographie en creux »21. Il a en effet montré que la géographie était utilisée dans le cours de Daubenton, dans celui de Thouin ; et que, les géographes eux-mêmes renvoyaient leurs élèves à d'autres cours. Ainsi, dans la première leçon, le 3 pluviôse an III (22 janvier 1795), après avoir énuméré tous les savoirs géographiques que le cours de mathématique, de physique générale et de chimie, d'histoire naturelle et d'histoire politique pourraient leur apporter, Buache de la Neuville déclara à ses élèves : «Vous concevez qu'il vous reste très peu de choses à faire pour apprendre ce qu'on appelle proprement la géographie. Les différents cours de l'Ecole normale vous en exposeront les circonstances fondamentales ; il ne sera plus question que d'y appliquer les détails. » 22.

$\mathrm{Au}$ sein du dispositif académique, cette fragmentation des savoirs géographiques entre plusieurs champs était aussi visible dans la dispersion de la géographie entre plusieurs académies. Alors que la géographie était représentée par un adjoint à l'Académie royale des sciences, il existait une classe de géographie ancienne au sein de l'Académie des Inscriptions et Belles Lettres. Ainsi, Bourguignon d'Anville entra d'abord en 1754 à l'Académie des Inscriptions et Belles Lettres, avant d'être élu à l'Académie des sciences en 177323. Un tel éparpillement de la géographie entre plusieurs institutions compliquait évidemment plus encore l'identification des animateurs de ce champ. Or, si, en 1795 lors de la mise en place de l'Institut, la création de la classe des sciences morales et politiques a offert une solution à cette fragmentation des savoirs géographiques, celleci ne s'est pas pérennisée et, dès 1803, la géographie a de nouveau été tiraillée entre les sciences et les belles lettres24. Cette fragmentation des savoirs géographiques apparait donc comme une figure durable de la géographie moderne, une figure certes datée, mais dont il faut mettre en lumière tous les enjeux.

\section{Les tentatives de définir la géographie}

Dès lors qu'un domaine de savoir est aussi fragmenté et qu'il ne peut se recommander d'aucune institution, comment parvenir à établir ce qui est géographique et qui sont les géographes ? Certains géographes se sont exprimés à ce propos. Ainsi, Malte-Brun considérait-il qu'un ouvrage

21 Daniel Nordman a eu la gentillesse de nous transmettre ce texte resté inédit et qu'il avait présenté en janvier 1995 lors du colloque intitulé «Autour de l'Ecole normale de l'an III ». Sa contribution s'attache à la géographie sans les géographes dans les cours de l'Ecole normale de l'an III.

22 NORDMAN (Daniel) (dir.), L'Ecole normale de l'an III., op.cit., p. 170.

23 BROC (Numa), La géographie des philosophes, op.cit., 20. 
Isabelle Laboulais, «Les paradoxes de la géographie des Lumières », dans Lise Andries (dir.), La construction des savoirs (XVIIIe-XIXe siècles), Lyon, Presses Universitaires de Lyon, 2009, p. 139-158.

pouvait être géographique «par le style ou par le savoir »25. Cependant, avec cette affirmation, plutôt que de clarifier les choses, Malte-Brun les a compliquées plus encore puisqu'il a retenu deux critères très généraux -le recours à la description et la production d'un savoir spatialisépour qualifier un texte de géographique. Cette conception n'était cependant pas originale; elle résultait certainement de la fusion des deux acceptions de la géographie que l'on retrouve aussi dans les définitions de ce terme rédigées pour les éditions successives du Dictionnaire de l'Académie française.

En 1694, dans la première édition, la géographie était simplement définie comme la « science qui enseigne la position de toutes les régions de la terre, les unes à l'égard des autres, \& par rapport au Ciel ». Mais, en 1798, dans la cinquième édition, la géographie était désormais définie par rapport à son objet, mais aussi par rapport à un type de discours, elle apparaissait donc comme la «Science qui enseigne la position de toutes les régions de la terre les unes à l'égard des autres, et par rapport au ciel, avec la description de ce qu'elles contiennent de principal ». A partir de 1798, la géographie ne fut plus seulement associée à la nomenclature mais à la description ; désormais, la définition était illustrée par des exemples comme «traité, cours de géographie, cartes de géographie », et complétée par la mention : «On dit de même, la géographie d'un pays, d'une province, etc. ». L'auteur de la notice du Dictionnaire de l'Académie française exprimait le lien entre cette forme discursive qu'est la description et cette catégorie de savoir qu'est la géographie. Cette intrication des deux notions transparaît aussi dans les notices du mot «description» proposées dans les différentes éditions du même dictionnaire. Dans la première édition, la description est définie comme un «discours par lequel on décrit, on dépeint » et aucun des exemples ne renvoie à la géographie ; en revanche en 1795 les exemples ont changé et l'on peut lire : «On appelle aussi description un livre qui contient l'état présent d'une province, d'un royaume, d'une partie du monde. Description de l'Egypte, de l'Afrique ». D'après ces définitions, il semble donc que la géographie se soit peu à peu démarquée de la simple nomenclature des positions pour devenir une description réunissant toutes les caractéristiques d'un pays ou d'une province. Si cette évolution répondait aux vœux de Mentelle qui déplorait «l'emploi trop

24 BLAIS (Hélène), « La géographie académique entre sciences et belles lettres (autour de la scission de 1803) », in Géographies plurielles..., op. cit., p. 95-111.

25 MALTE-BRUN (Conrad), Annales des voyages, op. cit., 1808, p. 6-7. 
Isabelle Laboulais, «Les paradoxes de la géographie des Lumières », dans Lise Andries (dir.), La construction des savoirs (XVIIIe-XIXe siècles), Lyon, Presses Universitaires de Lyon, 2009, p. 139-158.

rigoureux de ces méthodes abstraites qui donnent aux livres de géographie tant de sécheresse »26, elle ne permettait cependant pas d'identifier très nettement les compétences géographiques.

Après les notices des dictionnaires, si l'on se tourne vers les propos que les géographes euxmêmes ont tenus sur leur propre pratique, on s'aperçoit qu'au XVIIIe siècle, les compétences qu'ils associent à leur identité intellectuelle sont assez modestes. C'est du moins ce que semble suggérer Buache de la Neuville lorsqu'il affirme : «La géographie, au contraire, ne demande que des yeux et de la mémoire : ce qu'elle nous présente est réel et sensible, il n'est besoin ni de réflexion, ni de raisonnement pour le saisir; et toute personne, même un enfant en est capable »27, Lenglet-Dufresnoy n'était guère plus exigeant en 1712 lorsqu'il estimait que « La plupart des sciences demandent un esprit de réflexions qui sçache comparer les propositions les unes avec les autres. Mais la géographie, qui sert comme de principe aux connaissances les plus utiles dans l'usage de la vie, est une science des yeux, la mémoire n'y travaille que très peu, et même cette mémoire est locale, par l'application qu'elle fait de certains lieux à quelques parties d'un royaume ou d'une province »28. La géographie consiste seulement ici à produire une nomenclature précise. Masson de Morvilliers reprit cette conception de la géographie dans son discours en relevant : «Nous ne nous étendrons pas non plus sur la formation du globe, ni sur les différentes espèces de matières qui entrent dans sa composition, ou qui se rencontrent dans son intérieur. Le naturaliste les classe, le chimiste les analyse, le géographe doit se borner à indiquer les lieux où se trouvent ces substances »29. La géographie semble donc occuper une position subalterne puisqu'en s'assignant une tâche de localisation, elle limite le travail du géographe à réunir des données ; l'examen des causes des phénomènes revenant aux représentants des sciences les mieux constituées.

Ce partage des tâches qui semble réduire le rôle du géographe à la portion congrue pourrait, à première vue, passer pour de la résignation ; cependant les géographes revendiquaient ce rôle et soulignaient même son importance. Ainsi, déjà en 1755, dans son «Essai sur l'histoire de la géographie », Robert de Vaugondy distinguait les géographes des usurpateurs qu'il voyait comme

26 MENTELLE (Edme), Précis de la géographie universelle ou Description de toutes les parties du monde sur un plan nouveau, d'après les grandes divisions naturelles du globe, tome premier, Histoire de la géographie, Paris, 1810, p. 5.

27 BUACHE DE LA NEUVILLE (Jean-Nicolas), Géographie élémentaire moderne et ancienne contenant les principes de la géographie..., tome Ier, Paris, 1772, p. X.

28 LENGLET-DUFRESNOY, Méthode pour étudier la géographie dans laquelle on donne une description exacte de l'univers..., Tome Ier, Paris, 1716, p. I.

29 MASSON DE MORVILLIERS (Nicolas), op. cit., p. VII. 
Isabelle Laboulais, «Les paradoxes de la géographie des Lumières », dans Lise Andries (dir.), La construction des savoirs (XVIIIe-XIXe siècles), Lyon, Presses Universitaires de Lyon, 2009, p. 139-158.

« une foule d'ignorants dont la témérité et la présomption ont partagé l'attention des peuples, et surpris la confiance de ceux qui ne se sont pas tenus en garde : détournons nos regards de dessus eux, et ne les fixons que sur les dignes personnages qui par leurs travaux ont contribué aux progrès de la science dont j'ébauche l'histoire »30. Il insistait donc nettement sur la distinction entre les «sçavans qui ont composé des cartes particulières »31 et ceux dont le talent est de combiner et de discuter. Dans l'article «Géographie physique », Desmarest soulignait, lui, la nécessité de s'en rapporter aux témoignages des autres, pour autant il condamnait très vivement les compilateurs32. Lorsque les géographes s'expriment sur leur propre pratique, ils insistent sur leur capacité à réunir des savoirs divers, et soulignent que cette tâche ne consiste pas en une simple compilation, mais dans une combinaison de faits. Ainsi, comme le note Robert de Vaugondy, « l'astronome et le géomètre ont chacun des connaissances qui leur sont propres mais le géographe doit les posséder toutes, et être capable de discussion pour concilier et employer à propos les secours qu'il tire de l'un et de l'autre »33. On retrouve ici l'image de la géographie considérée comme une combinaison des découvertes faites par les disciplines spécifiques, comme un effort pour les organiser en une somme totale de l'expérience, bref la géographie comme discipline de synthèse. Or, derrière cette conception, la géographie est associée à l'histoire, Robert de Vaugondy note d'ailleurs : «Il est de la géographie comme de l'histoire ; celle-ci présente une suite de faits combinés et liés ensemble par un sçavant qui souvent n'en a pas été le témoin, mais qui ne décide de leur choix que par la connexion qu'ils ont entre eux »34. La construction des savoirs géographiques semble donc passer par des procédures qui relèvent des techniques classiques de l'érudition. Pour autant, à la fin du XVIIIe siècle, la méthode spécifiquement géographique semblait encore à définir. Ainsi, en 1808, Malte-Brun poursuivit l'inventaire de ces insatisfactions en relevant que «Cette activité irrégulière des géographes et des voyageurs gagnerait à se soumettre à une théorie et à une critique éclairée »35. En effet, faute de théorie, chacun pouvait se vanter d'être géographe. Les « géographes » constituaient donc un groupe très large, un groupe qui recouvrait en fait des cultures géographiques différentes et dont

30 ROBERT DE VAUGONDY (Didier), Essai sur l'histoire de la géographie, ou sur son origine, ses progrès et son état actuel, Paris, 1755, p. 230.

31 Idem, 158.

32 DESMAREST (Nicolas), « Géographie physique », in Encyclopédie ou dictionnaire raisonné des sciences, des arts et des métiers par une Société de gens de lettres, tome 7, Paris, 1757, p. 616.

33 ROBERT DE VAUGONDY (Didier), " Géographie », in Encyclopédie ou dictionnaire raisonné des sciences, des arts et des métiers par une Société de gens de lettres, tome 7, Paris, 1757, p. 613

34 ROBERT DE VAUGONDY (Didier), Essai sur l'histoire de la géographie, op. cit., p. 159. 
Isabelle Laboulais, «Les paradoxes de la géographie des Lumières », dans Lise Andries (dir.), La construction des savoirs (XVIIIe-XIXe siècles), Lyon, Presses Universitaires de Lyon, 2009, p. 139-158.

il faudrait mettre à jour la diversité des dispositifs de production de connaissances.

\section{Installer la géographie dans un horizon de savoir légitime}

Dans son «Discours sur la géographie», Masson de Morvilliers affirmait que la géographie « tient immédiatement à presque toutes les connaissances humaines » et que «pour y réussir avec quelque distinction, il faut être instruit dans les mathématiques et l'astronomie ; il faut connaître l'histoire de son pays et celle des autres peuples ; il faut avoir des idées saines sur la politique et le commerce, sur la physique, l'histoire naturelle et les arts. Il faut être en état enfin de comparer en philosophe les différentes mœurs des nations. »36. Or, cette manière de diluer la géographie n'est pas isolée, elle avait déjà été évoquée par Robert de Vaugondy dans son Histoire de la géographie et elle fut reprise, de manière analogue, par Malte-Brun dans son Précis de la géographie universelle37. Enfin, dans l'Encyclopédie de Diderot et d'Alembert, dans le discours préliminaire et dans le système figuré la géographie apparaissait aussi comme fille de l'astronomie et servante de l'histoire.

Paradoxalement donc, le fait que la géographie bénéficie de l'aura de sciences reconnues et que d'autres savants s'approprient ses objets et son registre discursif ne semble pas troubler un géographe autoproclamé comme Mentelle. A le lire, on a même plutôt l'impression que la géographie bénéficia du prestige de ces savoirs solidement établis. Il note ainsi : "Dans le commencement du XVIIIJe siècle, on persista encore à considérer la géographie comme une simple science auxiliaire, subordonnée à l'histoire. Cependant, la question de l'aplatissement du globe, objet de discussion entre Newton, Huyghens et Cassini valut à la géographie mathématique la protection des sociétés savantes, l'appui des gouvernements et une place parmi les sciences exactes.... »38. Nous avons déjà vu que la situation de la géographie n'était pas aussi valorisante au sein des institutions savantes, et particulièrement à l'Académie royale des sciences où il n'était pas question de géographie mathématique mais d'astronomie. Cependant, ce qu'il faut retenir ici, c'est l'assurance avec laquelle les géographes affirment les liens qu'entretient leur

35 MALTE-BRUN (Conrad), Annales des voyages, op. cit., 1808, p. 7.

36 MASSON DE MORVILLIERS (Nicolas), op.cit., p. V.

37 « nous nous sommes dit la géographie n'est-elle pas la sœur et l'émule de l'histoire ? Si l'une règne sur tous les siècles, l'autre n'embrasse-t-elle pas tous les lieux ? (...) Et cette description du globe n'est-elle pas intimement liée à l'étude de l'homme, à celle des mœurs, et des institutions ? N'offre-t-elle pas à toutes les sciences politiques des renseignements précieux ? Aux diverses branches d'histoire naturelle un complément nécessaire ? A la littérature un vaste trésor de sensations et d'images ? », MALTE-BRUN (Conrad), Précis de la géographie universelle, op. cit., p. $1-2$. 
Isabelle Laboulais, «Les paradoxes de la géographie des Lumières », dans Lise Andries (dir.), La construction des savoirs (XVIIIe-XIXe siècles), Lyon, Presses Universitaires de Lyon, 2009, p. 139-158.

discipline avec d'autres. Dans son Histoire des progrès de l'esprit humain dans les sciences exactes..., Saverien est particulièrement explicite quand il affirme que « l'astronomie et l'histoire sont les fondements de la géographie. La première fixe la position des lieux et l'histoire en donne la connaissance particulière $\gg 39$. Sa conviction n'est certes pas originale ; cependant, Saverien a le mérite de la clarté, il énonce clairement les deux vecteurs de légitimation, qui sont aussi les deux tutelles de la géographie.

L'astronomie apparaît à la fois comme une caution savante, mais aussi comme un passage obligé pour porter les résultats des observations de terrain, et plus particulièrement des navigations lointaines, dans un système de coordonnées. On peut ainsi lire sous la plume de Robert de Vaugondy : «L'on aurait encore retiré peu d'utilité de cette connaissance des parties du globe si l'on n'avoit usé des moyens sûrs pour en déterminer exactement la situation et la forme. »40. Qu'il s'agisse de la connaissance de la figure de la terre ou de la construction de la carte de France, ces tâches incombent toujours aux mathématiciens et le résultat de leurs travaux apparaît comme un préalable à celui des géographes. Ainsi, Gosselin, ne considérait-il pas ce lien de la géographie à l'astronomie comme une servitude. Au contraire, il estimait que l'astronomie avait permis de stabiliser les pratiques de la géographie, et notait ainsi : «Ortelius, par l'étendue et l'antériorité de ses travaux, doit être regardé comme le père de cette géographie historiquement prouvée, dans laquelle on fut obligé de se renfermer jusqu'à ce que l'Académie des sciences, au moyen des observations des astronomes envoyés dans les différentes parties du monde, eût créé et assis sur une base solide le système de la géographie moderne »41. C'est seulement ce détour par l'astronomie -puis par la géodésie- qui a permis aux géographes de dépasser le legs de Ptolémée pour déterminer à leur tour des listes de coordonnées fiables et associer ainsi leurs descriptions à des localisations plus sûres. De plus, l'astronomie a permis aux géographes de s'arroger un passé académique dont on a vu qu'il était pourtant peu prestigieux. Bien que marginalisés au sein de l'Académie des Sciences, les géographes s'appuyaient sur elle pour légitimer leur pratique. Un extrait de l'article «géographie» de Robert de Vaugondy est à cet égard assez explicite : «L'établissement de l'Académie donnant une face nouvelle aux connaissances mathématiques,

38 MENTELLE (Edme), Précis de la géographie universelle, op. cit., p. 523.

39 SAVERIEN, Histoire des progrès de l'esprit humain dans les sciences exactes et dans les arts qui en dépendent, Paris, 1765, p. 385.

40 ROBERT DE VAUGONDY (Didier), 1755, Essai sur l'histoire de la géographie, op. cit., p. I. 
Isabelle Laboulais, «Les paradoxes de la géographie des Lumières », dans Lise Andries (dir.), La construction des savoirs (XVIIIe-XIXe siècles), Lyon, Presses Universitaires de Lyon, 2009, p. 139-158.

releva infiniment la géographie par l'usage que l'on fit des observations astronomiques pour assigner aux parties de la terre les justes dimensions qu'elles doivent avoir. »42. L'astronomie, auxiliaire indispensable pour fixer la position des lieux et dresser la carte, apparaît donc aussi comme un moyen d'installer la géographie dans un horizon de savoir légitime et non plus dans l'ordre d'une connaissance spéculative du monde.

Dans la mesure où les années 1730-1740 sont marquées par l'émergence d'une éthique de l'exactitude et une transformation des pratiques de mesure 43 , l'astronomie apporte à la géographie cette caution qui devient nécessaire pour attester de la validité et de la pertinence d'un champ. Toutefois, Gosselin semble considérer que l'astronomie ne constitue pas la seule voie de légitimation pour la géographie. En évoquant les travaux de Bourguignon d'Anville, il cherche à montrer que la géographie ancienne peut, elle aussi, être porteuse de résultats stables. Il note : «Dans le siècle dernier, où les Leibniz, les Newton et leurs disciples avoient porté l'astronomie à son plus haut degré de perfection, où tous les arts et toutes les sciences des nations modernes sembloient en quelque sorte avoir été appelés au secours de la géographie, on vit le savant d'Anville, avec les seules mesures anciennes resserrer de près d'un tiers la largeur de l'Italie moderne, et ses résultats confirmés par toutes les opérations astronomiques qui ont été faites postérieurement »44. Aux yeux de Gosselin, la méthode de travail de l'historien semble aussi pouvoir être porteuse de légitimité.

Mais à la différence de la dépendance de la géographie vis-à-vis de l'astronomie, entre l'histoire et la géographie c'est, semble-t-il, plutôt une complémentarité qui s'est installée. La géographie apparaît comme indispensable pour l'histoire car, sans elle, il ne semble pas possible de fixer le cadre du récit. Ecoutons l'analyse qu'en propose Mahias : «Envisagée sous ce point de vue, l'étude de la géographie est donc d'une nécessité absolue, puisque sans les Lumières qu'elle répand sur l'histoire, il est impossible de mettre chaque Etat à sa place, d'en fixer les limites, d'en connaître les ressources, ce qui jette une confusion étrange dans le récit et dans la lecture des événemens, parce que ne pouvant les suivre exactement sur les lieux où ils se sont passés, il est

${ }_{41}$ GOSSELIN (Pascal F.J.), « Géographie ancienne », in DACIER (B.-J.), Rapports à l'Empereur sur le progrès des sciences, des lettres et des arts depuis 1789, Histoire et littérature ancienne, volume IV, Paris, Belin, 1989 (réédition), p. 196.

42 ROBERT DE VAUGONDY (Didier), Atlas universel, Paris, 1757, p. I.

43 BOURGUET (Marie-Noëlle), LICOPPE (Christian), « Voyages, mesures et instruments : une nouvelle expérience du monde au siècle des Lumières », Annales .E.S.C., 1997, n ${ }^{\circ}$ 5, p. 1149.

44 GOSSELIN (Pascal F.J.), « Géographie ancienne », op. cit., p. 192. 
Isabelle Laboulais, «Les paradoxes de la géographie des Lumières », dans Lise Andries (dir.), La construction des savoirs (XVIIIe-XIXe siècles), Lyon, Presses Universitaires de Lyon, 2009, p. 139-158.

impossible d'en saisir le fil et d'en apercevoir l'ensemble » 45. On retrouve ainsi de nombreuses explications qui reprennent le topos de la géographie considérée comme l'œil de l'histoire. Cette expression déjà utilisée par Ortelius dans son Theatrum orbis terrarum46 est également reprise dans l'Encyclopédie (dans le discours préliminaire mais pas dans le système figuré). Si la géographie est ainsi agrégée à l'histoire c'est parce que cette association semble permettre de poser les deux dimensions fondamentales de toute narration : le temps et l'espace. Cette association conduit parfois à les confondre, et comme le rappelle Malte-Brun, la géographie « ne diffère de l'histoire que parce que l'une se règle sur le temps et l'autre sur l'espace. La géographie n'est au fond qu'une histoire qui s'arrête pour considérer le présent $\gg 47$.

Jean-Marc Besse a récemment montré que si le rôle de la géographie consiste à mettre sous les yeux de l'historien ce qui est absent, cela ne vaut pas seulement pour l'histoire civile ou politique et pour les faits passés ; cela concerne aussi les phénomènes qui relèvent de l'histoire naturelle et qui sont observables sur toute la surface de la terre48. Avant le XIXe siècle, la géographie reste l'application par une quelconque science de la démarche qui vise à localiser un phénomène et à en décrire l'expression spatiale49. Ainsi les liens se tissent-ils peu à peu entre la géographie et tous les champs de savoirs qui tentent de rendre compte, et parfois d'expliquer, la spatialisation des phénomènes. C'est le cas notamment de l'économie politique, de la statistique ou des topographies médicales. Dans l'avertissement qui ouvre son dictionnaire d'économie politique, Démeuniez revendique ainsi la géographie politique comme préalable indispensable à son objet50. Pendant la Révolution et l'Empire, c'est une démarche assez similaire que l'on retrouve derrière tous les projets qui cherchent à dresser un inventaire des ressources de la République. Comme

45 MAHIAS (J.-M.), Géographie moderne de la France par le cours des fleuves et des rivières; ou méthode facile pour apprendre en peu de temps la nouvelle division..., 2 volumes, Paris, 1799, p. XXVI-XXVII.

46 «Mais de combien est nécessaire pour parvenir à cette intelligence des histoires, la connaissance des régions et provinces, et des mers, la situation des montagnes, des vallées, des villes, le cours des rivières, etc. (ce que des Grecs est appelé d'un nom propre Géographie, et d'aucuns gens doctes... l'œil de l'histoire) tous ceux là le confesseront facilement avec nous, qui y étant un peu moyennement instruits, ont aucune fois commencé à lire quelque histoire », cité par BESSE (Jean-Marc), Face au monde. Atlas, jardins, géoramas, Paris, Desclée de Brouwer, 2003, p. 107.

47 MALTE-BRUN (Conrad), Annales des voyages, op. cit., 1808, p. 5-6.

48 BESSE (Jean-Marc), Face au monde, op. cit., p.106.

49 OZOUF-MARIGNIER (Marie-Vic), "Géographie et histoire», in BAILLY (Antoine), FERRAS (Robert), PUMAIN (Denise) (dir.), Encyclopédie de géographie, Paris, Economica, 1992, p. 94.

50 «Il m'a semblé qu'à l'Economie politique, il falloit joindre la Géographie politique \& la Diplomatique oubliées dans l'ancienne Encyclopédie ; je les ai réuni à cet effet. Cette partie est donc absolument neuve ; elle contiendra plus de deux mille articles ; l'ancienne Encyclopédie n'en offre pas cent sur ces matières, \& il n'y en aura pas un seul de réimprimé mot à mot. On peut réduire à trois, les divisions générales du plan que je me suis formée ; $1^{\circ}$. la Géographie politique ; $2^{\circ}$ l'économie politique \& l'administration ; $3^{\circ}$. la diplomatique. », DEMEUNIER (Nicolas), 
Isabelle Laboulais, «Les paradoxes de la géographie des Lumières », dans Lise Andries (dir.), La construction des savoirs (XVIIIe-XIXe siècles), Lyon, Presses Universitaires de Lyon, 2009, p. 139-158.

dans la statistique départementale, l'espace reste ici un principe d'exposition, un moyen d'introduire ordre et liaison dans la description51. Le «Tableau des mines de la République » publié dans le Journal des mines en offre un bon exemple puisque chaque notice départementale commence par une description géographique. De même, lorsque le Journal des Arts et manufactures publie dans son premier numéro une lettre sur la « géographie industrielle », c'est pour annoncer la publication d'un ensemble de tableaux qui répertorie toutes les fabriques existant sur le territoire de la République et dressé par les soins de la Commission d'agriculture et des arts52.

Au tournant des XVIIIle et XIXe siècles, la géographie se trouve donc accaparée par d'autres champs et cette appropriation transparaît dans les manières de la désigner. La solution la plus courante pour désigner la géographie consiste à la subdiviser en géographies particulières qui répondent précisément aux besoins de ses différentes tutelles. Ce phénomène ancien ne fait que s'accentuer dans la première moitié du XIXe siècle. D'ailleurs, la définition du mot géographie proposée par l'édition de 1835 du Dictionnaire de l'Académie française précise qu'il est nécessaire d'indiquer « le point de vue particulier sous lequel on considère cette science », et sont alors énumérées la géographie ancienne, la géographie du Moyen Age, la géographie moderne, la géographie physique, la géographie astronomique, la géographie civile, historique politique. On retrouve donc là d'une part des subdivisions chronologiques, d'autre part des subdivisions thématiques, qui, les unes comme les autres, sont assez traditionnelles. On les retrouve en tout cas déjà dans l'Encyclopédie.

\section{Les subdivisions de la géographie}

Il semble qu'au XVIIIe siècle les subdivisions chronologiques de la géographie soient fixées selon des critères partagés par tous les auteurs. Tous les ouvrages consultés distinguent en effet la géographie ancienne, la géographie du Moyen Age et la géographie moderne. Robert de Vaugondy parle de trois âges différents qu'il définit ainsi : la géographie ancienne « qui est la description de la terre, conformément aux connoissances que les anciens en avoient jusqu'à la

Encyclopédie méthodique, Economie politique et diplomatique, partie présentée à Monseigneur le baron de Breteuil, ministre et secrétaire d'Etat... ,Tome premier, Paris - Panckoucke , Liège - Plompteux, 1784, p. III.

51 BOURGUET (Marie-Noëlle), Déchiffrer la France. La statistique départementale à l'époque napoléonienne, Paris, Editions des archives contemporaines, 1988, p. 84-87.

52 Journal des arts et manufactures. Publié sous la direction de la Commission exécutive d'agriculture et des arts, 1795 (an III). 
Isabelle Laboulais, «Les paradoxes de la géographie des Lumières », dans Lise Andries (dir.), La construction des savoirs (XVIIIe-XIXe siècles), Lyon, Presses Universitaires de Lyon, 2009, p. 139-158.

décadence de l'empire romain », la géographie du Moyen Age « depuis la décadence de l'empire jusqu'au renouvellement des Lettres », la géographie moderne «qui est la description actuelle de la terre, depuis le renouvellement des lettres jusqu'à présent $» 53$.

Dans cette répartition, le XVIe siècle est donc représenté comme un seuil épistémologique qui a fondé une pratique nouvelle à laquelle les géographes du XVIIIe siècle se rattachent encore. Ce seuil épistémologique est associé aux grandes découvertes qui ont laissé une part à l'expérience dans la construction des savoirs géographiques. Tous les auteurs reconnaissent que la géographie était cultivée dans l'Antiquité mais que la méthode leur faisait défaut. Ainsi, selon Mentelle « On ne peut disconvenir que les anciens manquaient de connaissances qu'ont employé les modernes pour la porter au degré de perfection où elle est arrivée de nos jours. Il fallait que d'un côté l'astronomie fît les progrès qu'elle doit aux belles découvertes qui nous ont fait connaître les satellites de Jupiter, et que, de l'autre, des hommes habiles correspondissent ensemble pour déterminer les longitudes avec précision »54. Et Malte-Brun d'ajouter, «N'oublions point que dans ce grand siècle les Copernic, les Tycho Brahé, et les Galilée perfectionnèrent cette science qui soumet les corps célestes aux calculs de l'homme (...) Dès lors, les énormes erreurs de Ptolémée, seul guide des géographes du moyen âge frappèrent les yeux. Il fallut absolument que la géographie changea de face $\gg 55$. C'est donc une double rupture que Malte-Brun suggère : d'une part, une rupture avec les textes anciens, d'autre part une rupture avec les géographes du Moyen Age qui, comme le souligne Santarem, ignoraient l'expérience du monde56. Masson de Morvilliers quant à lui rattache la rupture du XVIe siècle à la précision des connaissances géographiques et à l'articulation de l'astronomie et de la géographie. Dans son « Discours sur la géographie », il note que «la géographie ne peut guère remonter en France que jusque vers le milieu du seizième siècle, encore n'avons-nous eu que des essais informes qui se ressentaient de la barbarie des écoles et de nos faibles progrès dans la géométrie, l'astronomie et les autres sciences $» 57$. En affirmant : «C'est du temps de Mercator que date la géographie moderne $» 58$,

53 ROBERT DE VAUGONDY (Didier), « Géographie », op. cit., p. 613.

54 MENTELLE (Edme), Encyclopédie méthodique, Géographie ancienne, 3 volumes, Paris, Panckoucke, 1787, p. IV. 55 MALTE-BRUN (Conrad), Précis de la géographie universelle, op. cit., p. 521-522.

56 "Ces œuvres, antérieures au grand siècle des découvertes ont été exécutées dans des temps où la science géographique confondue au milieu des matières fort diverses, entrait dans le domaine de l'érudition, domaine alors plus que jamais fermé à l'expérience. Les érudits du moyen âge, malgré leur savoir, tenaient fort peu de compte des récits des voyageurs... », SANTAREM (Vicomte de), Essai sur l'histoire de la cosmographie et de la cartographie pendant le Moyen Age et sur les progrès de la géographie après les grandes découvertes du XVe siècle..., Paris, tome 2,1849 , p. XII.

57 MASSON DE MORVILLIERS (Nicolas), Encyclopédie méthodique, o. cit., p. XII. 
Isabelle Laboulais, «Les paradoxes de la géographie des Lumières », dans Lise Andries (dir.), La construction des savoirs (XVIIIe-XIXe siècles), Lyon, Presses Universitaires de Lyon, 2009, p. 139-158.

Malte-Brun, à son tour, synthétise ces deux positions. En effet, lorsque Mercator a édité la Géographie de Ptolémée, en 1578 puis en 1584, il a montré qu'une «distance définitive »59 existait entre lui et Ptolémée, ou plus exactement entre deux manières de cartographier le monde. Si les subdivisions chronologiques de la géographie sont assez bien fixées, en revanche, les subdivisions thématiques semblent plus incertaines. Ainsi, en 1722, lorsqu'il recensa les objets d'un bon dictionnaire géographique, Bruzen de la Martinière distinguait la géographie religieuse, la géographie politique, la géographie commerciale, la géographie mathématique et la géographie historique60 ; quarante ans plus tard, dans l'article « géographie » de l'Encyclopédie, Robert de Vaugondy proposait la division suivante : géographie astronomique, géographie naturelle, géographie historique, géographie civile ou politique, géographie sacrée, géographie ecclésiastique et géographie physique61; enfin Mentelle intitula l'un de ses ouvrages paru en 1803 Géographie mathématique, physique et politique de toutes les parties du monde, alors même que l'année suivante il publiait une Géographie physique, historique, statistique et topographique de la France. Même s'il s'agit tantôt d'une géographie générale, tantôt d'une géographie de la France, les catégories géographiques de Mentelle semblent bien fluides, d'autant que dans son « Tableau des différens rapports sous lesquels on peut considérer la géographie »62, il ne prend en compte que la géographie mathématique, la géographie physique et la géographie politique. Une fois identifiée la diversité de ces typologies, encore faut-il comparer les définitions données à chacune de ces branches de la géographie. C'est selon les objets sur lesquels la description s'arrête que la géographie prend des noms différents.

Bien que les désignations changent, on retrouve le plus souvent trois ensembles qui subdivisent et structurent les savoirs géographiques. Lorsqu'elle est liée à la fixation des positions, la géographie est dite mathématique ou astronomique, lorsqu'elle est liée à la description de la surface de la terre, la géographie est dite physique ou naturelle, lorsqu'elle est liée à l'histoire des états, elle est dite géographie politique ou civile. Les tutelles classiques sont donc ici réaffirmées et organisées ou superposées comme s'il fallait d'abord situer un lieu à la surface de la terre, puis

58 MALTE-BRUN (Conrad), Précis de la géographie universelle, op. cit., p. 522.

${ }_{59}$ Cité par BESSE (Jean-Marc), Les grandeurs de la terre. Essai sur les transformations du savoir géographique à la Renaissance, Lyon, ENS Editions, 2003, p. 23.

60 BRUZEN DE LA MARTINIERE, «Essai sur l'origine et les progrès de la géographie depuis la découverte de l'Amérique jusqu'à présent avec des remarques sur les principaux géographes modernes », Mémoires historiques et critiques, tome second, Amsterdam, J.-F. Bernard, 1722, p. 40.

61 ROBERT DE VAUGONDY (Didier), « Géographie », op. cit., p. 608.

62 MENTELLE (Edme), Encyclopédie méthodique,op. cit. 
Isabelle Laboulais, «Les paradoxes de la géographie des Lumières », dans Lise Andries (dir.), La construction des savoirs (XVIIIe-XIXe siècles), Lyon, Presses Universitaires de Lyon, 2009, p. 139-158.

dégager son environnement, avant de s'attacher aux actions humaines. Toutefois, au début du XIXe siècle, à mesure que de nouvelles disciplines se sont constituées, de nouvelles formes de géographies, ou du moins de nouvelles désignations sont apparues. Citons la géographie ethnographique parfois désignée comme géo-ethnographie63, ou la géographie botanique64, ou encore la géographie zoologique. Ces deux derniers exemples témoignent de la manière dont la géographie s'est vue appropriée par l'histoire naturelle. Dans le volume 7 du Dictionnaire classique d'histoire naturelle qui fut publié sous la direction de Bory de Saint-Vincent en 1825, l'article «Géographie sous les rapports de l'histoire naturelle » était divisé en deux sections, la «Distribution géographique des productions aquatiques » et la «Distribution géographique des productions terrestres». Cette dernière était subdivisée en «géographie botanique» et « géographie zoologique »65. Parmi ces nouvelles désignations de la géographie, la « géographie physique » occupe une place particulière car, si l'expression est ancienne, elle semble, à la fin du XVIIIe siècle, être réinvestie d'un sens nouveau. Au tout début de l'article «géographie physique» publié en 1757 dans l'Encyclopédie, Desmarest définit la géographie physique comme une «description raisonnée des grands phénomènes de la terre, et la considération des résultats généraux déduits des observations locales et particulières, combinées et réunies méthodiquement sous différentes classes et dans un plan capable de faire voir l'économie naturelle du globe, en tant qu'on l'envisage seulement comme une masse qui n'est ni habitée, ni féconde »66. L'originalité du propos ne réside pas ici dans l'usage de l'expression « géographie physique », mais plutôt dans l'acception qui est revendiquée. Par rapport aux définitions les plus courantes de la géographie, Desmarest change d'objet, il abandonne les Etats pour s'attacher aux phénomènes de la terre, il écarte aussi l'homme et la société de ses préoccupations. Par rapport aux définitions courantes de la géographie physique, il exclut les bassins fluviaux pourtant considérés comme l'apanage de cette forme de géographie depuis 1752 et l' «Essai de

63 BRAVO (Michael T.), «Ethnographic Navigation and the Geographical Gift", in LIVINGSTONE (David N.), WITHERS (Charles W. J.) (eds), Geography and Enlightenment, Chicago, The University of Chicago Press, 1999; DIAS (Nella), Une science nouvelle ? La géo-ethnographie de Jomard », in BOURGUET (Marie-Noëlle), LEPETIT (Bernard), NORDMAN (Daniel), SINARELLIS (Maroula) (dir.), L'invention scientifique de la Méditerranée. Egypte, Morée, Algérie, Paris, EHESS, 1998, p. 159-183.

64 DROUIN (Jean.-Marc), «Bory de Saint-Vincent et la géographie botanique », in BOURGUET (Marie-Noëlle), LEPETIT (Bernard), NORDMAN (Daniel), SINARELLIS (Maroula) (dir.), L'invention scientifique de la Méditerranée, op. cit., p. 139-157.

65 Ibid., p. 142.

66 DESMAREST (Nicolas), « Géographie physique », op. cit., p. 613. 
Isabelle Laboulais, «Les paradoxes de la géographie des Lumières », dans Lise Andries (dir.), La construction des savoirs (XVIIIe-XIXe siècles), Lyon, Presses Universitaires de Lyon, 2009, p. 139-158.

géographie physique » de Buache67.

Cependant, le dernier volume de l'Encyclopédie méthodique qui, après la mort de Desmarest, fut achevé par Bory de Saint-Vincent et Huot montre bien que cette volonté n'a pu s'imposer face à la géologie qui, au cours des premières décennies du XIXe siècle s'est constituée en véritable discipline. En 1828, Huot témoigne d'ailleurs de cette évolution : «Depuis l'année 1811, que le quatrième volume de cet ouvrage a paru, les explorations ont continué partout avec le même zèle ; les observations physiques se sont multipliées ; les recherches géologiques, qu'il est impossible, selon nous, de ne pas rattacher à la géographie-physique, ont pris une direction nouvelle, et de nombreuses observations ont ajouté à son importance et à l'intérêt qu'elles présentoient déjà depuis long-temps »68. C'est ainsi qu'il mit un terme à la tentative de Desmarest de faire de la géographie physique un domaine propre et qu'il imposa la géologie, réduisant de facto le champ de la géographie à la production de savoirs localisés.

Plutôt que de valoriser la géographie, le poids de l'étymologie, qui fait d'elle la description de la terre, l'a condamnée à une position difficilement tenable. Pour faire face à cette définition, ce domaine de savoir devait ou bien s'arroger un statut méta-théorique ou bien se résigner à une position ancillaire. Le statut méta-théorique aurait consisté à ce que la géographie serve de socle à la réflexion des domaines de savoir qui regardent l'espace comme un principe d'intelligibilité, un peu à la manière de ce qu'Humboldt imagina dans son ouvrage Cosmos paru en 1846. Au contraire, le statut ancillaire restreint la tâche de la géographie à la production d'une nomenclature. Celle-ci apparaît comme la forme première du discours géographique, elle permet de répondre à la question « où ? », et produit ainsi des données qui peuvent être utilisées par d'autres.

Cette hétérogénéité de la géographie académique ne doit pas occulter la géographie pratiquée. Cela dit, pour saisir ce dont cette géographie-là était faite, il faut sortir du corpus propre à la discipline et retrouver ses héritages multiples qui ne relèvent pas seulement de l'univers de la

67 Dans l'Encyclopédie méthodique, Masson de Morvilliers reconnaît lui aussi la singularité des objets de la géographie physique, et lui attribue même des outils spécifiques : les bassins fluviaux de Buache, «ce sont, écrit-il, autant de bassins particuliers qui appartiennent à chaque pays et que la géographie physique doit d'abord faire connaître », MASSON DE MORVILLIERS (Nicolas), op. cit., p. VIII.

68 HUOT (J.N.), Encyclopédie méthodique. Géographie-physique par M. Desmarest, Continuée par MM. Bory de Saint-Vincent, de la même Académie; Doin, docteur en médecine ; Ferry, l'un des rédacteurs de la revue encyclopédique; Huot, de la Société d'histoire naturelle de Paris et de plusieurs autres sociétés savantes. Tome cinquième, Paris, Agasse, 1828, p.857. 
Isabelle Laboulais, «Les paradoxes de la géographie des Lumières », dans Lise Andries (dir.), La construction des savoirs (XVIIIe-XIXe siècles), Lyon, Presses Universitaires de Lyon, 2009, p. 139-158.

science mais aussi de l'univers de la pratique69. Dès lors, comment constituer le corpus d'étude ? Certes, des travaux ont déjà été menés sur les ingénieurs des Ponts et Chaussées, sur les officiers du Génie ou sur les administrateurs, des groupes qui, chacun à leur manière, tentent de rendre intelligible le territoire où s'exerce leur autorité. Comment cependant identifier tous les discours qui prennent en charge les représentations spatiales ? Comment localiser dans le champ de la pratique la diversité des savoirs spatiaux souvent dénués de reconnaissance académique ? Il s'agit là de questions essentielles si l'on veut comprendre l'identité des géographes ainsi que les conditions de possibilité d'un savoir relatif à l'espace.

69 «Tout comportement prend place dans l'espace mais n'a pas nécessairement l'espace pour objet ou enjeu. Il vaut mieux réserver le terme d'action spatiale aux actions dont le but consiste à modifier le rapport d'un sujet à l'espace (comme les déplacements) », STASKAK (Jean-François), « Pratique spatiale », in LEVY (Jacques), LUSSAULT (Michel) (dir.), Dictionnaire de la géographie et de l'espace des sociétés, Paris, Belin, 2003, p. 741. 